\title{
A new experimental route in thermomechanics of inorganic glasses using infrared thermography
}

\author{
Jean-Benoît Le Cam ${ }^{\text {a,* }}$, Eric Robin ${ }^{\mathrm{a}}$, Xavier Balandraud ${ }^{\mathrm{b}, \mathrm{c}}$, Evelyne Toussaint ${ }^{\mathrm{c}, \mathrm{d}}$ \\ a Université De Rennes 1, L.A.R.M.A.U.R-CNRS 6274, Campus de Beaulieu, Bât. 10B, 35042 Rennes Cedex, France \\ b Clermont Université, Institut Français de Mécanique Avancée, Institut Pascal, BP 10448, 63000 Clermont-Ferrand, France \\ c CNRS, UMR 6602, Institut Pascal, 63171 Aubière, France \\ d Clermont Université, Université Blaise Pascal, Institut Pascal, BP 10448, 63000 Clermont-Ferrand, France
}

\begin{abstract}
A theoretical approach based on thermomechanics of irreversible processes shows that the heat sources produced by a soda lime glass specimen under cyclic mechanical loading lead to temperature variations which can be detectable with a high resolution infrared camera. In order to validate this approach, experimental investigations are performed. For this purpose, a cyclic bending test is carried out and infrared measurements are performed. Temperature variations measured at the specimen surface are found to reach a few hundredths of a degree for a stress amplitude of the order of magnitude of $40 \mathrm{MPa}$. Results are in a good agreement with theoretical predictions. These thermal measurements are then used for thermoelastic stress analysis and heat source calculation. The study demonstrates that such an experimental approach based on infrared thermography will improve the understanding of the thermomechanical behavior of inorganic glasses.
\end{abstract}

\section{Introduction}

Thermal and calorimetric effects accompanying the deformation of materials are widely studied in literature to investigate thermomechanical couplings, fatigue and failure, non-exhaustively. Most of applications deal with metals, but polymers, ceramics and composite materials are also studied. Concerning inorganic glass materials, studies generally focus on crack tip movement [1], mechanical properties [2] or fracture $[3,4]$. To the best knowledge of the authors, the thermal response has never been considered to investigate the mechanical behavior of inorganic glasses. The objective of the present work is therefore to evidence thermal and calorimetric effects in a soda lime glass subjected to cyclic loading at room temperature. A high resolution infrared (IR) thermography and a suitable post-processing of the thermal signal are used for this purpose.

IR thermography provides accurate information about temperature changes occurring on the surface of specimens. These changes in temperature can be due to different phenomena: thermoelasticity, change in material phase, viscosity, fatigue damage, etc. Thermal effects and more precisely calorimetric effects associated with the mechanisms of deformation have been largely studied with this type of technique. The approach consists in estimating the heat sources produced by the material from the temperature fields obtained with an IR camera and the heat diffusion equation (see for instance [5-8]). In the framework of thermodynamics of irreversible processes
(TIP), such heat source determination can be used to establish or validate constitutive equations.

The aim of the present study is therefore to determine if such an experimental approach can be applied to inorganic oxide glass materials. A first question is to know if temperature variations due to elastic deformation can be experimentally detected. A second question is to know if heat sources can be accurately estimated from temperature measurements. This paper is composed of two sections. Section 2 deals with the general thermomechanical framework that allows us to predict the thermal and calorimetric responses of the soda lime glass under uniaxial mechanical loading. Section 3 presents the experimental investigations carried out and the discussion on the results obtained during a bending test.

\section{Prediction of the thermal response of soda lime glass under mechanical loading}

\subsection{A brief recall of the thermomechanical background}

Any thermomechanical system out of equilibrium is considered as the sum of several homogeneous subsystems at equilibrium. In this context, constitutive equations are established by considering the deformation process as issued from a quasi-static thermodynamic process. This process can be mechanically dissipative. Assuming the Fourier's law for the heat conduction, the heat diffusion equation writes:

$\rho C \frac{\partial T}{\partial t}-k \operatorname{div}(\operatorname{grad} T)=s_{h}+r_{e x t}$

\footnotetext{
* Corresponding author. Tel.: + 33223235741; fax: + 33223236111.

E-mail address: jean-benoit.lecam@univ-rennes1.fr (J.-B. Le Cam).
} 
where $T$ is the temperature, $r_{\text {ext }}$ the external heat source and $s_{h}$ the heat source produced or absorbed by the material itself. The material parameters are the density $\rho$, the thermal conductivity $k$ and the specific heat $C$ (assumed to remain constant during the deformation process). Temperature variations $\theta=T-T_{\text {ref }}$ are defined with respect to a reference temperature field $T_{\text {ref, }}$ which is defined when no heat source is produced by the specimen. If the external heat source $r_{e x t}$ is assumed to not vary over the test duration, Eq. (1) becomes:

$\rho C \frac{\partial \theta}{\partial t}-k \Delta \theta=s_{h}$

For a thermoelastic behaviour, the heat source $s_{h}$ produced by the material is composed of the thermoelastic (isentropic) coupling term, denoted $s_{\text {the }}$ in the following, and possibly the mechanical (intrinsic) dissipation $d_{1}$. The latter is related to mechanical irreversibilities occurring in the material. This quantity is always positive. For glass materials, which are assumed to behave as isotropic thermoelastic materials, the quantity $s_{\text {the }}$ is written $-\alpha T_{0} \frac{\partial \operatorname{tr}(\sigma)}{\partial t}$, where $\alpha$ is the coefficient of thermal expansion, $T_{0}$ the mean temperature of the specimen over the test (in Kelvin) and $\operatorname{tr}(\sigma)$ the trace (sum of principal stresses) of the Cauchy stress tensor $\sigma$. Thus, Eq. (2) comes:

$\rho C \frac{\partial \theta}{\partial t}-k \Delta \theta=-\alpha T_{0} \frac{\partial \operatorname{tr}(\sigma)}{\partial t}+d_{1}$.

Eq. (3) shows that a cyclic mechanical loading leads to a temperature evolution composed of a global increase (due to mechanical dissipation) and an oscillation at the same frequency as the load but in opposite phase (due to the thermoelastic coupling). If the loading frequency is sufficiently high, adiabatic conditions are achieved over one cycle, and consequently the conduction term can be neglected. As mechanical dissipation due to fatigue can be considered as negligible over one cycle, the amplitude of temperature oscillation $\Delta \theta$ during one cycle is directly proportional to the amplitude $\Delta \operatorname{tr}(\sigma)$ of the trace of the Cauchy stress tensor:

$\Delta \theta=-A \Delta \operatorname{tr}(\sigma)$

where $A$ is equal to $\frac{\alpha T_{0}}{\rho C}$. This gives rise to numerous applications in the field of the thermoelastic stress analysis. The reader can refer to $[9,10]$ for further information.

\subsection{Prediction of the temperature variation at the surface of a thin glass specimen}

This section summarizes assumptions used to simplify the heat diffusion equation in the case of homogeneous deformation fields.

Let us consider a specimen geometry and a mechanical loading such that the deformation field is homogeneous in the specimen at any time. Due to the very small thermal diffusivity of glasses (order of magnitude of $10^{-7} \mathrm{~m}^{2} \cdot \mathrm{s}^{-1}$, see Table 1) compared to metals for instance, the temperature field is almost homogeneous in the specimen at any time. As a consequence, the temperature at the surface is nearly equal to the mean temperature over the volume. The heat diffusion equation can be written in this case $[8,11-13]$ :

$\rho C\left(\frac{\partial \theta}{\partial t}+\frac{\theta}{\tau}\right)=s_{\text {the }}$

where $\tau$ is a time constant characterizing the global heat exchanges between the specimen and its surroundings. The value of $\tau$ characterizes the level of non-adiabaticity of the thermodynamical evolution. For instance, if $\tau$ is much larger than the test duration, the evolution can be considered as adiabatic. In the case of a thin specimen, $\tau$ is nearly equal to $\frac{e \rho C}{2 h}$, where $e$ is the thickness and $h$ the coefficient of convection with ambient air. This formulation of the heat diffusion equation
Table 1

Thermophysical properties of a sodalime glass.

\begin{tabular}{ll}
\hline Properties & Value \\
\hline Young modulus $E(\mathrm{GPa}), 25{ }^{\circ} \mathrm{C}$ & 70 \\
Poisson's ratio $\nu(-), 25{ }^{\circ} \mathrm{C}$ & 0.22 \\
Density $\rho\left(\mathrm{kg} / \mathrm{m}^{3}\right), 25{ }^{\circ} \mathrm{C}$ & 2500 \\
Specific heat $C(\mathrm{~J} /(\mathrm{kg} \mathrm{K})), 25{ }^{\circ} \mathrm{C}$ & 800 \\
Thermal diffusivity $D\left(\mathrm{~m}^{2} \mathrm{~s}^{-1}\right), 25{ }^{\circ} \mathrm{C}$ & $3 \times 10^{-7}$ \\
Coefficient of thermal expansion $\alpha\left(\mathrm{K}^{-1}\right), 5-35{ }^{\circ} \mathrm{C}$ & $9 \times 10^{-6}$ \\
Thermoelastic coefficient $A=\frac{\alpha T_{0}}{\rho C}(\mathrm{~K} / \mathrm{MPa}), 25^{\circ} \mathrm{C}$ & $1.32 \times 10^{-3}$ \\
\hline
\end{tabular}

can be referred to as the "OD" formulation. The reader can refer to [5-7] for further information.

The present study focuses on the thermoelastic coupling. In the case of isotropic thermoelastic materials under uniaxial loading condition, the heat source $s_{\text {the }}$ is also equal to $-\alpha T_{0} E \dot{\epsilon}$ where $\varepsilon$ is the axial strain and $E$ the elastic modulus. The sign of the heat source when the mechanical loading oscillates is the opposite of the strain rate one. It should be noted that heat source only depends on the strain rate. Under triangular signal, the strain rate and consequently the heat source are constant. This is a feature of purely isentropic thermoelastic material. Finally, the expression of the heat source also shows that the heat source is not constant when the strain rate is not, which is often the case for experimental conditions. This is why sinusoidal signals are considered in the following to predict the heat source and temperature variations.

\subsection{Numerical predictions}

Numerical predictions are carried out with the following conditions:

- the mechanical loading consists of tensile cycles in such a way that the uniaxial strain oscillates between 0 and $5 \times 10^{-4}$, which corresponds to a stress variation between 0 and $35 \mathrm{MPa}$;

- a sinusoidal signal shape is considered;

- the frequency is set to $3 \mathrm{~Hz}$;

- thermophysical properties, issued from the Sciglass database [14] are reported in Table 1.

Under adiabatic conditions, the frequency does not influence the amplitude of the temperature oscillation. Under non-adiabatic conditions, it affects the temperature variation because of the heat exchanges with the outside. In the experiments presented in Section $3, \tau$ is equal to
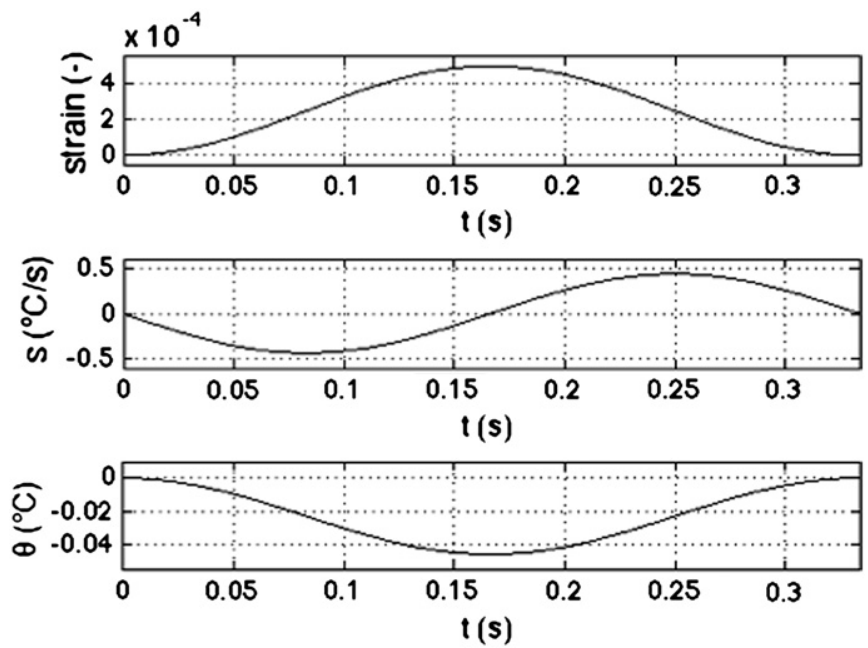

Fig. 1. Strain, heat source and temperature variation during one adiabatic mechanical cycle at $3 \mathrm{~Hz}$. The corresponding stress amplitude is $35 \mathrm{MPa}$. 


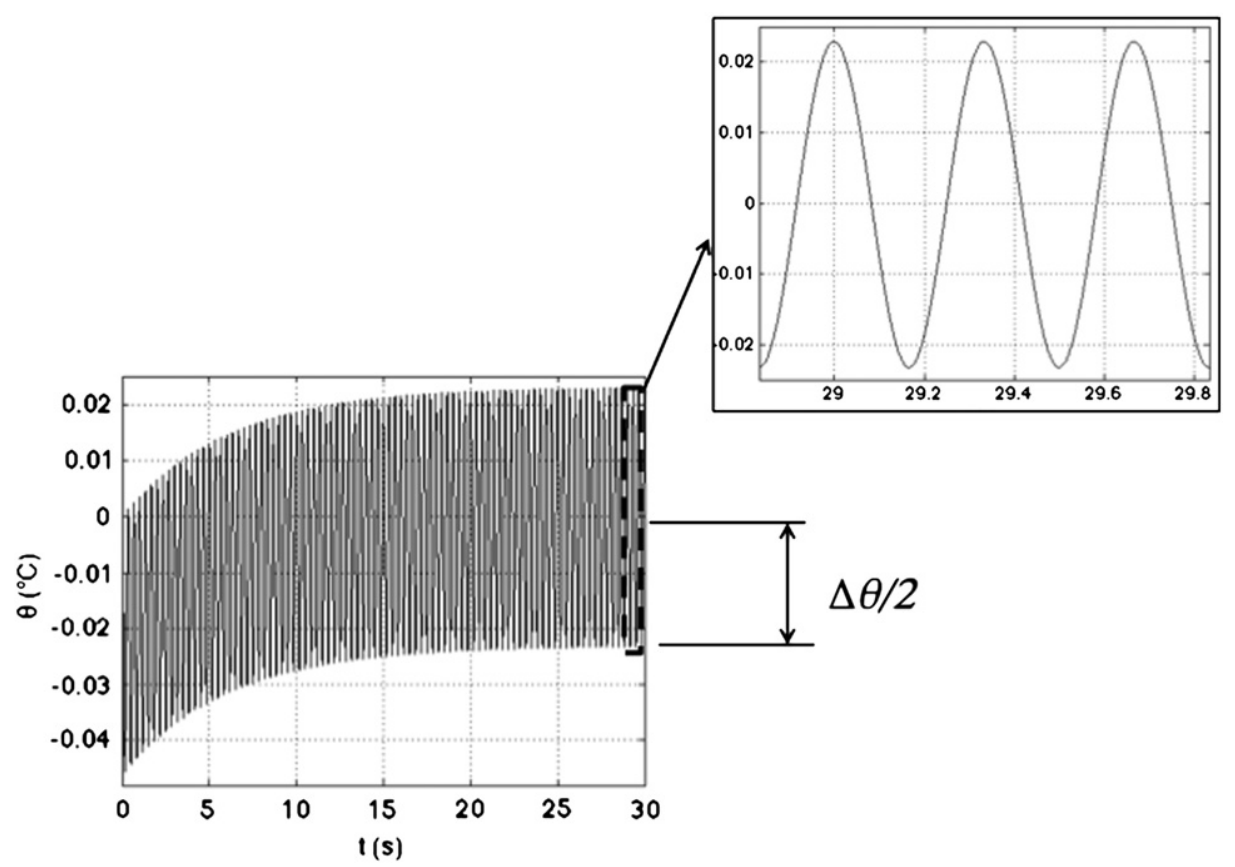

Fig. 2. Evolution of the temperature variation up to thermal stabilization during mechanical cycles at $3 \mathrm{~Hz}$.

$130 \mathrm{~s}$. This means that the steady-state regime of the temperature evolution is reached from about 3 or 4 times this duration. For the numerical applications below, the time constant $\tau$ is reduced to $6 s$ and the test duration is set to $30 \mathrm{~s}$. This enables us to observe more clearly the transient and steady-state regimes. Besides, this reduces the calculation time.

The heat source $s_{\text {the }}$ is expressed in $\mathrm{W} \mathrm{m}^{-3}$. However, it is useful to divide this quantity by $\rho C$, leading to a unit in ${ }^{\circ} \mathrm{C} \mathrm{s}^{-1}$. For the sake of simplicity, this latter unit will be used below for the "heat source" $s=s_{\text {the }} / \rho C$. Using this unit enables us to obtain the temperature rate in case of adiabatic evolution. In case of non-adiabatic conditions, the temperature rate is different, due to the heat exchange with the outside.

Results are presented in Fig. 1. In this figure, from the top to the bottom, the evolutions of strain, heat source and temperature variation in adiabatic conditions are given for one mechanical cycle. As expected, the temperature decreases when the strain increases, and inversely. Fig. 2 presents the evolution of the temperature variation during the test in non-adiabatic conditions. It starts with a temperature decrease as the test starts by a strain (stress) increase, before reaching the steady-state regime. The main result is that the temperature variations can reach a few hundredths of a degree and consequently that such temperature variations are detectable by using a high resolution IR camera. Experimental results are presented in the next section.

\section{Experimental investigations}

The previous section has evidenced that the temperature variations during tensile loading should be detectable with a high resolution infrared camera. Due to the difficulties for applying cyclic tensile loading conditions to glass materials, bending tests are preferred. It should be noted that bending test induces heterogeneous strain and stress states, including both tension and compression, which provides more relevant information for validating thermomechanical modeling.

\subsection{Mechanical test and measurements}

The specimen is $20 \mathrm{~mm}$ in length, $5 \mathrm{~mm}$ in width and $3 \mathrm{~mm}$ in thickness. It was tested under cyclic bending loading by means of a $15 \mathrm{kN}$ MTS testing machine. The experimental setup is presented in Fig. 3. The applied loading was sinusoidal and the load ratio $R$, defined as the ratio between the minimum and the maximum values of the force applied, was equal to 0.1 for all the tests. Table 2 gives the seven tests carried out. $F_{\text {mean }}$ and $F_{a m p l}$ are the mean value and the amplitude
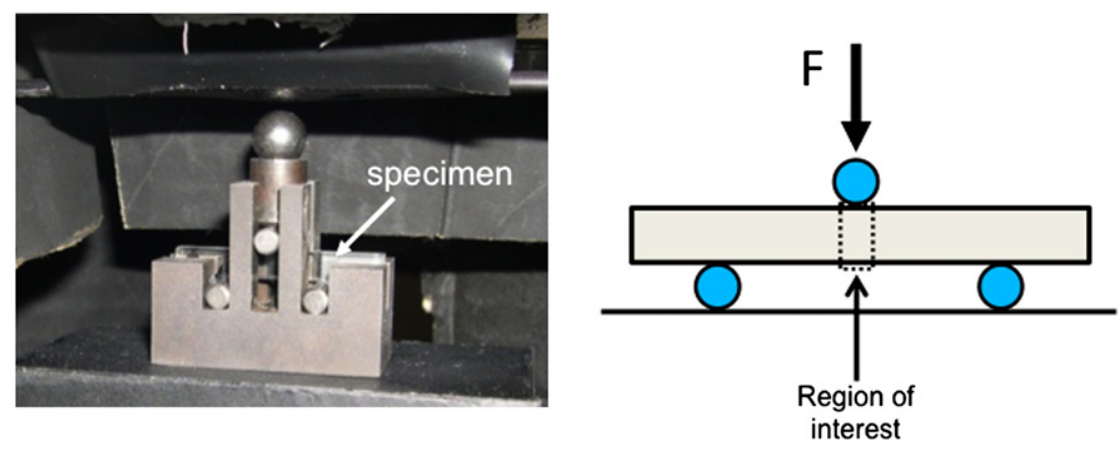

Fig. 3. Experimental setup. 
Table 2

Mechanical tests performed.

\begin{tabular}{llll}
\hline Test number & $F_{\text {mean }}(N)$ & $F_{\text {ampl }}(N)$ & $f_{L}(\mathrm{~Hz})$ \\
\hline 1 & -27.5 & -22.5 & 1 \\
2 & -55 & -45 & 1 \\
3 & -82.5 & -67.5 & 1 \\
4 & -110 & -90 & 1 \\
5 & -27.5 & -22.5 & 3 \\
6 & -55 & -45 & 3 \\
7 & -82.5 & -67.5 & 3 \\
\hline
\end{tabular}

of the force applied, respectively. $f_{L}$ is the loading frequency. Two frequencies were applied, 1 and $3 \mathrm{~Hz}$.

Because of the low thermal diffusivity $D$ of the material compared to metals for instance, loading frequencies $f_{L}$ superior to $1 \mathrm{~Hz}$ are assumed to lead to adiabatic conditions within the specimen. Indeed, the thermal diffusion length, defined as $\sqrt{D /\left(\pi f_{L}\right)}$ [15] for $f_{L}=1 \mathrm{~Hz}$ is equal to $0.31 \mathrm{~mm}$. This length can be assumed lower than the stress spatial gradient.

Temperature measurements were performed at ambient temperature using a Cedip Jade III-MWIR infrared camera which features a local plane array of $320 \times 240$ pixels and detectors with a wavelength range of 3.5$5 \mu \mathrm{m}$. The integration time is $1500 \mu \mathrm{s}$ and the acquisition frequency is $147 \mathrm{~Hz}$. The resolution is equal to about $20 \mathrm{mK}$ in the $\left[5^{\circ} \mathrm{C} ; 40^{\circ} \mathrm{C}\right]$ temperature range. For temperature variations of a few degrees, the resolution is equal to $20 \mathrm{mK}$. The thermal emissivity of the material is close to 1 (>0.84). The spatial resolution, corresponding here to the size of the pixel on the specimen, is equal to $0.089 \mathrm{~mm}$. In order to ensure that the internal temperature of the camera is stabilized for performing the measurements, it was switched on for $4 \mathrm{~h}$ before the experiment. The temperature measurements were performed during $10 \mathrm{~s}$ for each test.

\section{Results and discussion}

Fig. 4 gives the temperature variation range (the peak-to-peak amplitude) on the observed surface for tests \#1 to \#7. The values of amplitude between maximum and minimum temperature variations are calculated over one period. Such maps allow us to evaluate the level of the temperature variation amplitude according to the cyclic loading applied. Besides, they allow us to identify the zones of highest thermal activity for a given cyclic loading condition. The highest loading condition applied at $1 \mathrm{~Hz}$ is not presented for $3 \mathrm{~Hz}$ due to the specimen failure. The results show that the order of magnitude of the temperature variation range is a hundredth of a degree, which corroborates the prediction of the theoretical approach presented in Section 2. Moreover, the mappings show that the highest the distance to the neutral axis, the highest the temperature amplitude. This is in agreement with the mechanical states induced by the bending test. It can be noted that the contact between the specimen and the roll induces a stress concentration in the bulk material below the contact zone as predicted by the Hertz theory [16].

In order to more precisely discuss on the temperature variation range according to the pixel location, Fig. 5 gives the evolution of the temperature variation $\theta$ measured at three points on the specimen surface for test \#4. One point is located in a compression zone in the influence of the contact condition (point $\mathrm{H}$ ). The second point is located on the neutral axis (point $\mathrm{N}$ ). The third one is located in the tension zone (point $\mathrm{L}$ ). Temperature variations at points $\mathrm{L}$ and $\mathrm{H}$ oscillate in opposite phase. This is in good agreement with the fact that one point is in a tension zone and the other one in a compression zone. The amplitude of the temperature variations is equal to about $0.05{ }^{\circ} \mathrm{C}$ and to $0.10{ }^{\circ} \mathrm{C}$ at points $\mathrm{L}$ and $\mathrm{H}$, respectively. These results also highlight that no temperature variation is detected at the neutral axis, which corroborates the beam theory [17]. At point $\mathrm{H}$, the amplitude is higher due to the contact between the specimen and the roll.
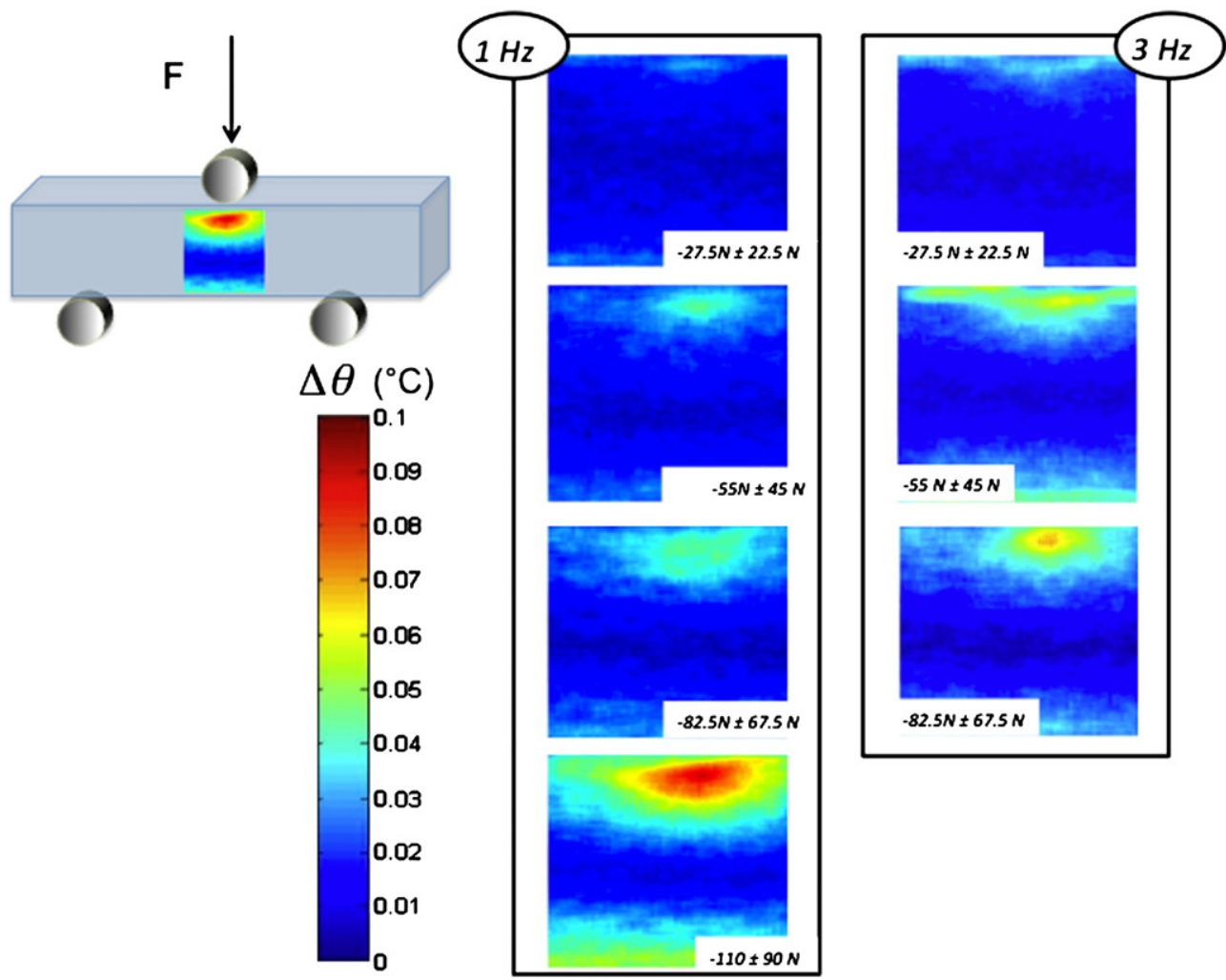

Fig. 4. Maps of temperature variation range for tests \#1 to \#7. 

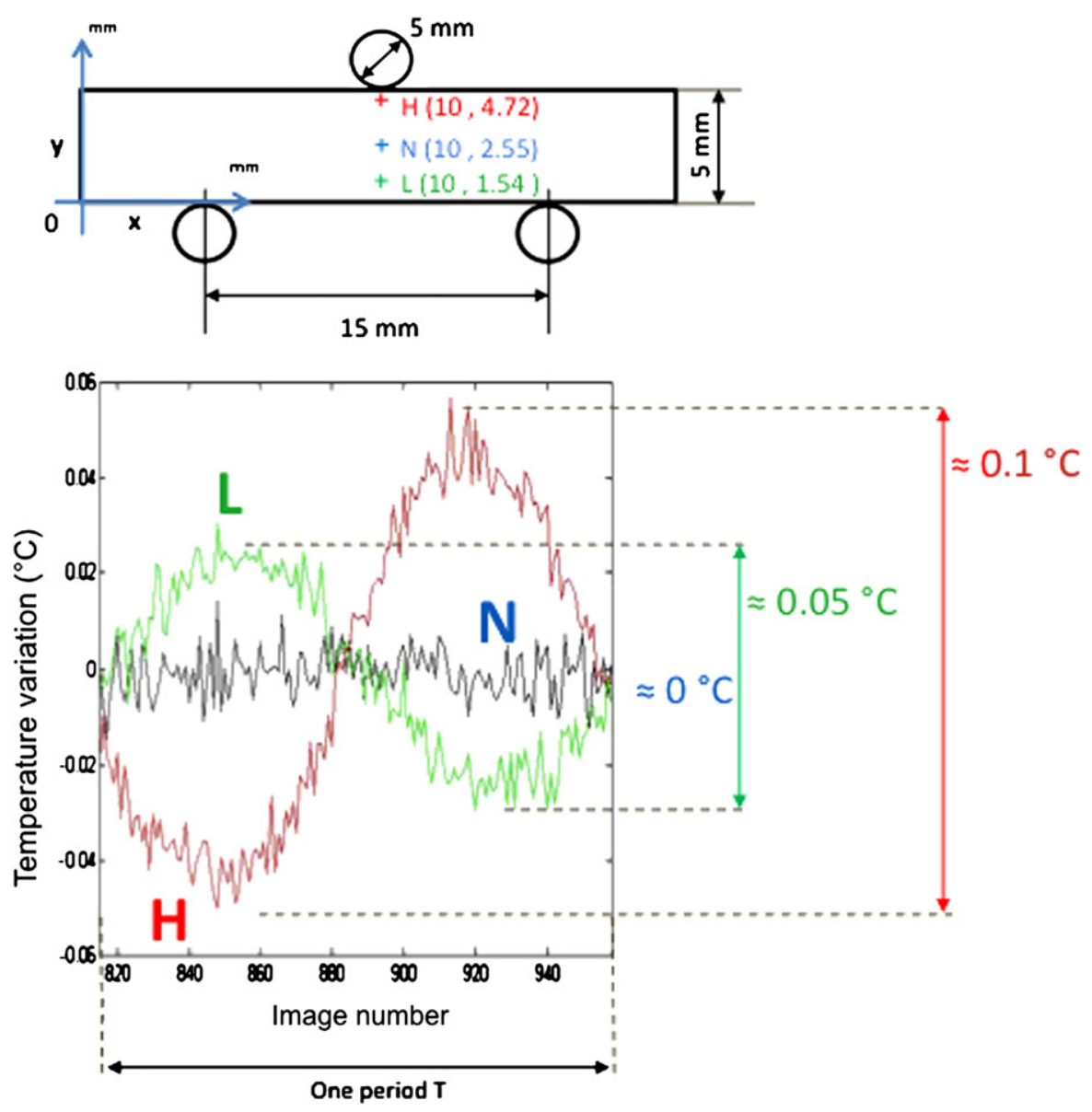

Fig. 5. Evolution of the temperature variation at three different points on the specimen surface (test \#4).

These first results evidence that an inorganic oxide glass material produces and absorbs heat during its deformation, leading to temperature variation which is detectable with a high sensitive infrared camera. This proves that quantitative calorimetric analysis can be carried out with this type of material. As an example, at point $\mathrm{L}$, the temperature variation obtained under adiabatic-like condition $\left(0.05{ }^{\circ} \mathrm{C}\right)$ corresponds to predicted uniaxial strain and stress levels under adiabatic condition of about $5 \times 10^{-4}$ (see Fig. 1 ) and $35 \mathrm{MPa}$, respectively.

Next paragraphs present quantitative analyses of the thermal measurements, i.e. thermoelastic stress analysis and heat source calculation.

Fig. 6 gives the map of the temperature variation amplitude measured at the specimen surface for test \#4. Contrarily to previous maps,

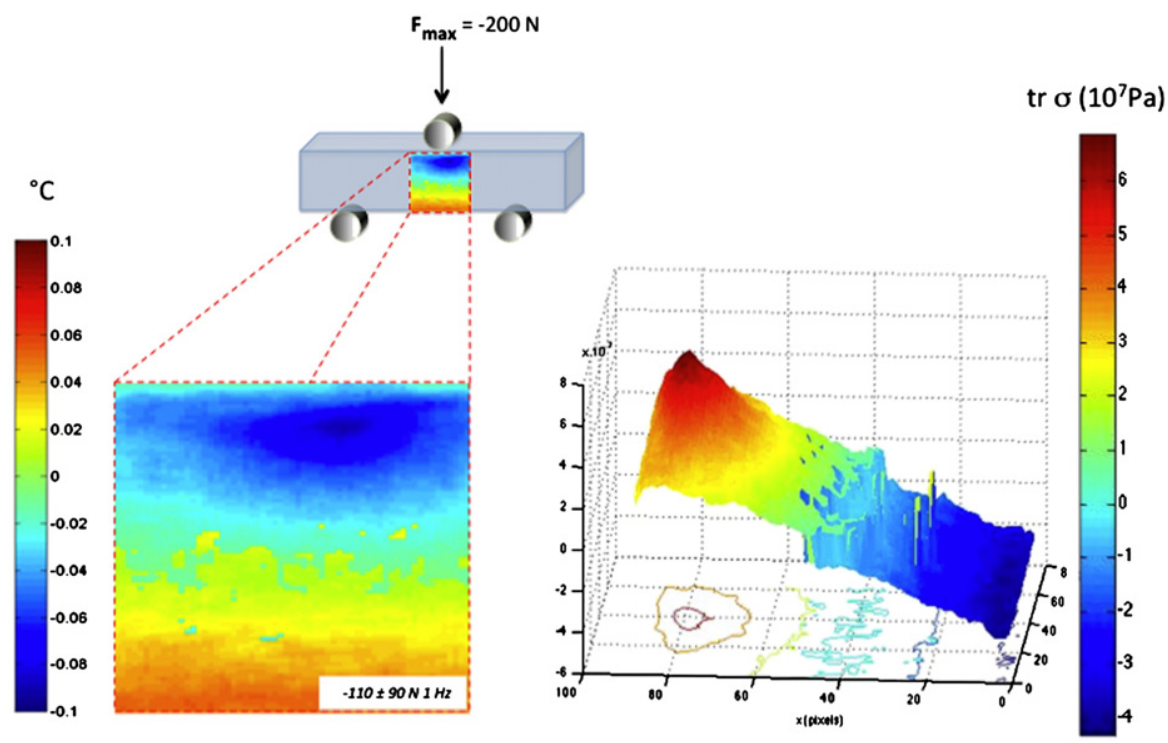

Fig. 6. Temperature variation field at the specimen surface (left-hand side). Corresponding field in terms of stress (on the right-hand-side). 


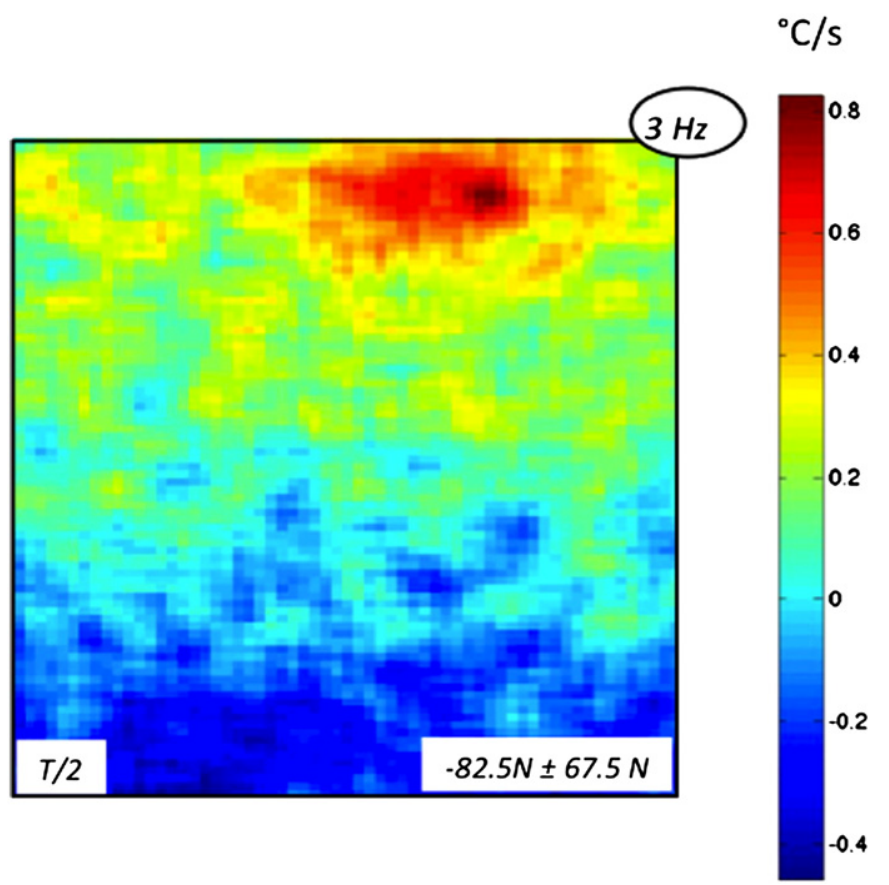

Fig. 7. Maximum heat source at each point during test \#7.

the field is signed by determining the phase shift between the temporal temperature variations. This field is then used in order to link the temperature amplitude to the trace $\operatorname{tr}(\sigma)$ of the Cauchy stress tensor by means of Eq. (4). Thus, the diagram at the bottom of the figure presents the mapping of $\operatorname{tr}(\sigma)$ on the observed surface. The maximum of $\operatorname{tr}(\sigma)$ is obtained below the contact zone due to the contact between the roll and the specimen. Except in this zone, the stress field can be considered as uniaxial, consequently $\operatorname{tr}(\sigma)$ gives the value of the Cauchy stress in the specimen length direction. According to the beam theory, the stress is of opposite sign on both sides of the neutral axis and its maximum value equates $\sigma_{\max }=\frac{3 F L}{2 b h^{2}}(60 \mathrm{MPa}$ in the present case). This is in very good agreement with the experimental results obtained from the temperature variation field measurement. This illustration of the possibilities offered by using a thermoelastic stress analysis shows that stresses can be accurately measured in inorganic glasses.
Fig. 7 shows the heat source field (maximum over the cycle) calculated from temperature variation field by using Eq. (5). As the test conditions are quasi-adiabatic, the heat source and the temperature variation fields are similar and are in good agreement with the prediction obtained by assuming the material to be isotropic and thermoelastic.

\section{Conclusion}

A theoretical analysis has shown that thermoelastic heat sources in inorganic oxide glass during its deformation are detectable by a high resolution infrared camera. Moreover, we have shown that thermal measurements can be analyzed quantitatively in order to carry out thermoelastic stress analysis and to calculate heat sources absorbed and produced by the material according to the considered change in the mechanical state. This allows us to envisage to link calorimetric effects accompanying the deformation of inorganic glasses with changes in their structure and to improve the understanding of their thermomechanical behavior.

\section{Acknowledgments}

Authors thank the financial support by "Rennes Metropole" and the "Mission Ressources et Compétences Technologiques" (MRCT) group of the National Scientific Research Council (CNRS).

\section{References}

[1] R. Weichert, K. Schönert, J. Mech. Phys. Solids 26 (1978) 151-161.

[2] C. Pouvreau, M. Drissi-Habti, K. Michel, B. Bureau, J.-C. Sangleboeuf, C. BoussardPledel, T. Rouxel, J.-L. Adam, J. Non-Cryst. Solids 316 (2003) 131-137.

[3] M.V.Swain, J.C. Metras, C.G. Guillemet, J. Non-Cryst. Solids 38-39 (1980) 445-450, (Part 1).

[4] J.-P. Guin, S.M. Wiederhorn, J. Non-Cryst. Solids 316 (2003) 12-20.

[5] A. Chrysochoos, H. Louche, Int. J. Eng. Sci. 38 (2000) 1759-1788.

[6] A. Chrysochoos, H. Louche, Mater. Sci. Eng., A 307 (2001) 15-22.

[7] X. Balandraud, A. Chrysochoos, S. Leclercq, R. Peyroux, C.R. Acad. Sci. Ser. IIb: Mec. Phys. Chim. Astron. 329 (2001) 621-626.

[8] B. Berthel, A. Chrysochoos, B. Wattrisse, A. Galtier, Exp. Mech. 48 (2008) 79-90.

[9] J.M. Dulieu-Barton, E.G. Little, J. Eaton-Evans, I.A. Brown, Strain 44 (2007) 102-118.

[10] N. Sathon, J.M. Dulieu-Barton, Appl. Mech. Mater. 7 (8) (2007) 153-158.

[11] A. Chrysochoos, H. Pham, O. Maisonneuve, Nucl. Eng. Des. 162 (1996) 1-12.

[12] X. Balandraud, E. Ernst, E. Soos, C.R. Acad. Sci. Ser. IIb: Mec. Phys. Chim. Astron. 327 (1999) 33-39.

[13] T. Boulanger, A. Chrysochoos, C. Mabru, A. Galtier, Int. J. Fatigue 26 (2004) 221-229.

[14] SciGlass 7.7, http://www.sciglass.info/ 2012.

[15] S. Offermann, J.-L. Beaudoin, C. Bissieux, H. Frick, Exp. Mech. 37 (1997) 409-413.

[16] H.J. Hertz, Reine, Angew. Math. 92 (1882) 156-171.

[17] S.P. Timoshenko, Theory of Elastic Stability, McGraw-Hill, New York, 1961. 